\title{
Effectiveness of pharmacist intervention for asthma control improvement
}

\author{
E. Mehuys*, L. Van Bortel" ${ }^{\#}$ L. De Bolle*, I. Van Tongelen*, L. Annemans", \\ J.P. Remon* and G. Brusselle
}

ABSTRACT: Education on optimal medication use is an essential strategy to improve asthma control. The current authors investigated whether pharmacist interventions, focused on appropriate use of asthma medication and tailor-made to the patient's current asthma control, would improve asthma control in adult patients.

A 6-month randomised, controlled, parallel-group trial was conducted in 66 community pharmacies in Belgium. Patients were randomly assigned to receive usual pharmacist care $(n=94)$ or a pre-defined pharmacist intervention $(n=107)$. This intervention mainly focused on improving inhalation technique and medication adherence. Primary outcome was the level of asthma control, as assessed by the Asthma Control Test ${ }_{\mathbb{R}}$ (ACT).

Mean ACT scores did not change from baseline for both study groups. However, a pre-defined subgroup analysis of patients having insufficiently controlled asthma at baseline showed that the intervention had significantly increased the ACT score after 6 months compared with usual care. The intervention also reduced, for the complete study group, reliever medication use and the frequency of night-time awakenings due to asthma. Inhalation technique and adherence to controller medication were significantly better in the intervention group.

In conclusion, pragmatic community pharmacy-based programmes can significantly improve therapeutic outcomes in adult asthma patients.

KEYWORDS: Adherence, asthma, asthma education, inhalation technique, pharmacotherapy

$\mathbf{T}$ he aim of asthma management is to gain and maintain control of the disease (defined as "asthma control"). Although clinical trials have shown that good asthma control can be achieved in a majority of patients [1], this is not the case in real-life situation studies [2]. A large number of patients have not yet benefited from the advances in asthma treatment and are still insufficiently controlled, placing severe limits on daily life and putting them at risk for asthma-related morbidity and mortality. In an attempt to improve asthma control, the Global Initiative for Asthma (GINA) recently updated its asthma management guidelines and now emphasises asthma management based on clinical control (GINA 2006) [3] rather than on asthma severity (GINA 1995 and 2002) [4, 5]. This is an important paradigm shift for asthma care and implies that the level of asthma control should be continuously monitored (due to the variable nature of the disease) and that treatment should be adjusted according to the patients' current asthma-control status (controlled, partly controlled or uncontrolled).

Nowadays, asthma management programmes are mainly delivered in a hospital setting and/or by physicians. However, community pharmacists could also make a useful contribution to asthma management due to their expertise on medication and their frequent contacts with the patient on prescription refill. Pharmacists could assist asthma patients and their physicians to achieve and maintain asthma control by providing the patient with suitable information and training about asthma medication, instructing correct inhalation technique, questioning the patient's understanding of the role of their asthma medications, explaining why inhaled corticosteroids are necessary, addressing the patient's concerns about potential side-effects of inhaled corticosteroids and facilitating adherence to controller medication. These factors have already been shown to be an important barrier to the achievement of good asthma control, as prescribing an appropriate asthma control treatment may not be successful when the patient uses the medication inappropriately [6-9]. Only a few well-designed studies have investigated the effect of pharmacist care on asthma patients to date [10-15]. These studies have shown improvements in peak flow [12-15], asthma severity [10, 13], symptom scores [11], drug utilisation $[10,12-14]$ and humanistic
AFFILIATIONS

*Pharmaceutical Care Unit Ghent,

Faculty of Pharmaceutical Sciences,

${ }^{\#}$ Heymans Institute of Pharmacology, Depts of "Public Health, Faculty of Medicine and Health Sciences, and ${ }^{+}$Respiratory Diseases, Ghent University Hospital, Ghent, Belgium.

CORRESPONDENCE

E. Mehuys

Pharmaceutical Care Unit Ghent

Faculty of Pharmaceutical Sciences

Ghent University

Harelbekestraat 72

9000 Ghent

Belgium

Fax: 3292228236

E-mail: els.mehuys@ugent.be

Received:

August 272007

Accepted after revision:

November 282007

STATEMENT OF INTEREST

A statement of interest for this study

can be found at www.erj.

ersjournals.com/misc/

statements.shtml 
outcomes (e.g. quality of life, asthma knowledge) [10, 12-14]. However, the pharmacist interventions described in these publications [10-15] were not tailored to the patients' current level of asthma control (as recommended by GINA 2006). The evaluated interventions also focused on several aspects of asthma management, as follows. 1) Choice of drug therapy: by identifying and solving drug-related problems, for example, by referral to a physician for adding a drug, for a dosage change, for a change of dosage form, etc. 2) Appropriate use of the medication: inhalation technique and adherence. 3) Selfmanagement: stimulating patients towards more self-management, for example, by goal setting. Such "broad" pharmacist interventions are not only time-consuming (e.g. SAINI et al. [13] recorded that the total mean time spent per patient was $96.4 \mathrm{~min}$ across three visits) but also require extensive education and training of the pharmacists (e.g. learning how to interpret peak flow measurements). Time constraints and lack of education have already been identified as important barriers to the implementation of pharmaceutical care into community pharmacy practice in Europe [16].

Furthermore, none of the previous studies used asthma control as the main clinical outcome measure. They mainly used peak expiratory flow or asthma severity as the primary outcome. Several of these studies investigated only certain aspects of asthma control (e.g. daily dose of salbutamol based on dispensed medication history [10] or Medical Research Council dyspnoea scale [12]). However, current GINA guidelines recommend evaluating all aspects of asthma control, which is necessary to gain a complete view of the patients' asthma-control level, with a clinically validated measure.

For these reasons, the current authors designed a feasible intervention focused only on ensuring that patients use their prescribed drug therapy in the correct way (i.e. correct inhalation technique and good medication adherence). To ensure that the intervention agreed with the new GINA guidelines, the Asthma Control Test ${ }_{\circledR}$ (ACT; Quality Metric, Lincoln, RI, USA) was used as a rapid and easy tool to determine the level of asthma control of patients presenting at community pharmacies and to provide targeted pharmacist advice (table 1) [17]. The present randomised controlled trial was set up to study the hypothesis that such pharmacist intervention, focused on the optimal use of asthma medication and tailor-made to the patient's current asthma control, would result in an improved asthma control in adult patients over a 6-month period. Primary outcome was the level of asthma control, as measured by the ACT. Secondary outcomes included the patient's peak expiratory flow, rescue medication use, night-time awakenings due to asthma, inhalation technique, adherence to controller medication, severe exacerbations, quality of life, knowledge on asthma and smoking behaviour.

\section{METHODS}

\section{Patients}

Asthma patients were recruited consecutively in 66 randomly selected pharmacies, located in diverse areas of Flanders, the Dutch-speaking northern part of Belgium. To be eligible, patients were required to carry a prescription for asthma medication (R03, Anatomical Therapeutic Chemical classification). In consecutive order, patients visiting the pharmacy were invited to participate in the study when they fulfilled the

\section{TABLE 1 Overview of pharmacist intervention}

Session 1: at start of intervention period

Personal education from the pharmacist about the following topics: Correct use of the inhaler device

Understanding asthma*

Symptoms

Triggers

Early warnings

Understanding asthma medication

Difference between controller and reliever medication

Facilitate adherence to controller medication

Smoking cessation (if relevant)

Sessions 2 and 3: at 1-month and 3-month follow-up, respectively

Pharmacist advice based on the ACT score of the patient:

If ACT score <15 ( "uncontrolled" asthma): immediate referral to general practitioner or respiratory specialist

If ACT score 15-19 ("insufficiently controlled" asthma): review inhalation technique and check controller medication adherence

If ACT score $\geqslant 20$ ("well-controlled" asthma): no specific advice needed, inform patient asthma is well controlled

ACT: Asthma Control Testæ (QualityMetric, Lincoln, RI, USA). * : using the Dutch version of the Global Initiative for Asthma Patient Guide "What You and Your Family Can Do About Asthma" [18].

following inclusion criteria: 1) aged between 18-50 yrs; 2) being treated for asthma for $\geqslant 12$ months; 3) "using" controller medication; and 4) regular visitor to the pharmacy. Exclusion criteria included a smoking history of $>10$ pack-yrs, suffering from another severe disease (e.g. cancer) and having an ACT score at screening of $<15$ (indicating seriously uncontrolled asthma; for ethical reasons, these patients were immediately referred to their general practitioner (GP) or respiratory specialist) or equalling 25 (indicating complete asthma control; no room for improvement).

\section{Study design}

This 6-month randomised, controlled, parallel group trial was carried out between January 2006 and October 2006 (patient recruitment period: January-April 2006). The study was performed in accordance with the Declaration of Helsinki and Good Clinical Practice guidelines. Approval for the study was granted by the ethics committee of the Ghent University Hospital, Ghent, Belgium. All patients gave written informed consent. The GP of each participant was informed about the study by letter.

The study had a 2-week run-in period, followed by 6 months of randomised treatment. There were five scheduled visits to the pharmacy as follows: at the start of the run-in period, at randomisation and at 1, 3 and 6 months after randomisation.

All patients entering the run-in phase had to keep an asthma diary for 2 weeks (see section on Diary data). At the end of the run-in phase, patients were eligible for randomisation if they returned to the pharmacy with a diary that was completed for $\geqslant 90 \%$. Eligible patients were randomly assigned to either the control group or the intervention group. The sequence of allocation to either control or intervention group was 


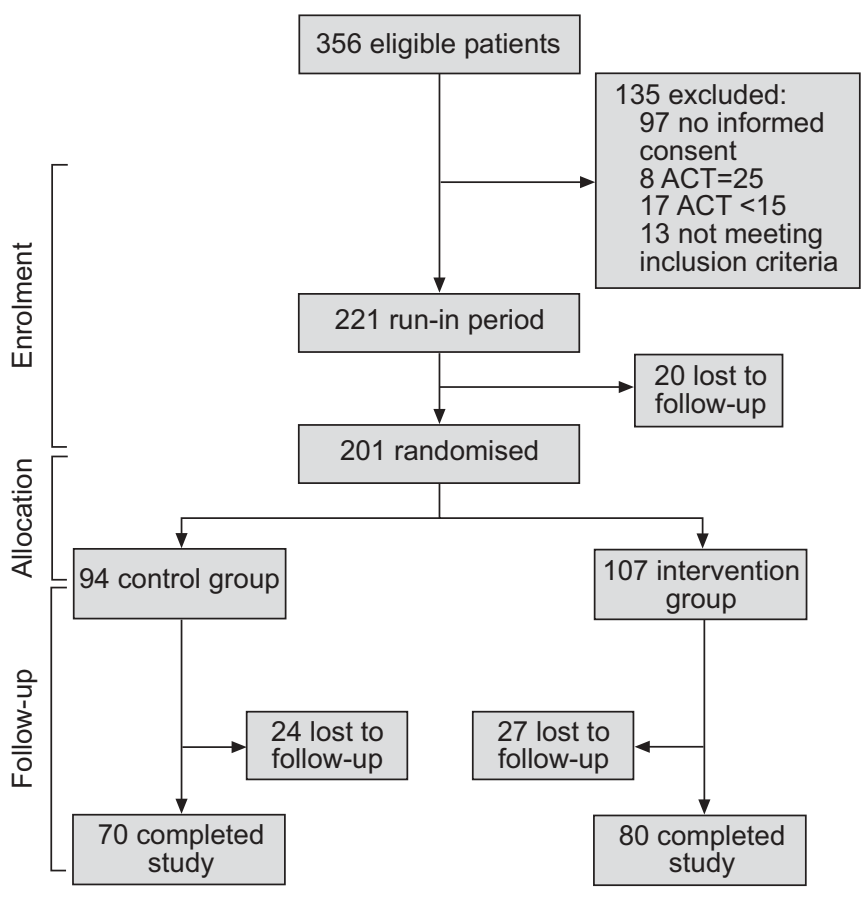

FIGURE 1. Flow of participants through the study. ACT: Asthma Control Test $\pi$ (QualityMetric, Lincoln, RI, USA).

predetermined by the investigators based on a randomisation table. Serially numbered, closed envelopes were made for each participating pharmacy. The envelope with the lowest number was opened by the pharmacist upon inclusion of a new patient.

\section{Intervention}

Before the start of the present study, the participating pharmacists had a training session about asthma (pathophysiology), its nonpharmacological and pharmacological treatment (GINA guidelines) and about the use of the study protocol.

Patients in the intervention group received a protocol-defined intervention at the start of the study and at the 1- and 3-month follow-up visits (fig. 1). Patients in the control group received usual pharmacist care.

\section{Outcomes}

Asthma control

The primary outcome was the level of asthma control, which was measured with the ACT (Dutch version). This is a clinically validated measure for asthma control, consisting of five questions, each having five possible response modalities (classified by decreasing level of asthma control, scored from 5 to 1) $[19,20]$. The ACT score (range 5-25) was determined by summing the response scores to the five questions; the higher the score, the better the asthma control. Patients with a maximal score of 25 were considered "totally controlled"; an ACT score of 20-24 indicated "well-controlled" asthma; an ACT score of 15-19 indicated "insufficiently controlled" asthma; a score $<15$ indicated "uncontrolled" asthma.
The ACT was filled out by patients at the start of the run-in phase, at randomisation and 1, 3 and 6 months after randomisation.

\section{Diary data}

Patients filled in a daily diary during the 2-week run-in phase, after 3 months (during weeks 11 and 12) and after 6 months (during weeks 23 and 24) treatment. The following information was recorded in the diary: 1) nocturnal awakenings due to asthma; 2) the number of inhalations of rescue medication (during the day or night); and 3) the best of three measurements of peak expiratory flow (PEF) made with a Mini-Wright Standard Peak Flow Meter (Clement Clarke, Harlow, UK) in the morning and evening before medication. PEF data are expressed as the percentage of maximum predicted value based on the patient's sex, age and height [21].

During the entire study period, patients also registered asthmarelated emergency department visits and hospitalisations.

\section{Severe exacerbations of asthma}

A severe exacerbation of asthma was defined as one requiring treatment with oral glucocorticoids (individually recorded in computerised pharmacy records) or an emergency department visit or hospital admission due to asthma.

\section{Adherence to controller medication}

Adherence during the course of the study was assessed using two validated measures: prescription refill rates and selfreporting [22]. The number of units of controller medication dispensed to each patient during the study period was available through computerised pharmacy records. The daily dose prescribed was read from the prescription or asked of the patient. From these data, adherence to controller medication was calculated and expressed as follows [23]:

adherence rate $(\%)=($ total number of days supplied during the study-number of days of last supply in the study)/(last claim

date in the study-first claim date in the study) $\times 100$

Self-reported adherence was assessed at the end of the study by asking the patients: "How often do you not take your controller medication as prescribed? (a) Never, (b) 1-2 times $\mathrm{yr}^{-1}$, (c) 1-2 times'month ${ }^{-1}$, (d) 1-2 times'week ${ }^{-1}$, (e) daily."

Asthma-specific quality of life

Asthma-specific quality of life was assessed at the start of the intervention period and after the 6-month follow-up period using the Dutch version of the Standardised Asthma Quality of Life Questionnaire (AQLQ(S)) according to JUNIPER et al. [24].

\section{Knowledge about asthma and treatment}

Patients' knowledge about asthma and its treatment was evaluated at the start of the intervention period and after the 6-month follow-up using an updated version of the Knowledge of Asthma and Asthma Medicine questionnaire [25].

\section{Inhalation technique}

The inhalation technique was scored using a checklist (eightpoint checklist for metered dose inhalers (MDI), 10-point checklist for MDI used with a large volume spacer and eightpoint checklist for dry powder inhalers (DPI)) at the start of the intervention period and 6 months later. For each correct step, one point was assigned and the sum score of the inhalation 
technique was displayed as percentage of correct steps. Patients committing major errors in inhalation technique (for MDI, failure to remove cap and/or fail to shake MDI; for DPI, failure to load device and/or fail to inhale quickly and deeply through device) were assigned a sum score of zero. For ethical reasons, such major errors were also corrected in patients belonging to the control group.

\section{Statistical analysis}

Sample size was calculated based on the ability to detect a $10 \%$ difference in the ACT score between the intervention group and the control group with $80 \%$ power at the $5 \%$ significance level. For an estimated $30 \%$ attrition, $\sim 200$ patients were enrolled into the present study.

The success of randomisation was assessed by comparing baseline characteristics of both study groups using unpaired t-tests for continuous variables and Chi-squared tests for categorical variables. These tests were also used to compare baseline characteristics of patients who did and did not complete the study.

The primary outcome, i.e. the ACT score, was analysed using an intention-to-treat approach. A linear mixed model was used, with the maximum-likelihood method used to handle missing data [26]. To account for any cluster effect (i.e. correlation of patients within pharmacies), a multilevel logistic regression was performed on the primary outcome. Pharmacies were used as level-one observations, and patients as level-two observations. The intra-pharmacy correlation coefficient was shown to be very small $(<0.1)$, meaning there was no significant cluster effect. A priori, it seemed likely that mainly the insufficiently controlled patients (ACT score $<20$ ) would benefit the most from intervention. Therefore, before the start of the study, the current authors decided to perform a subgroup analysis of patients with insufficient control on the primary outcome measure, the ACT.

The secondary outcomes were analysed on a per-protocol approach. The continuous parameters measured at baseline, and at 3 and 6 months were analysed using a repeatedmeasures multivariate ANOVA with baseline values as covariates. Treatments by time interactions were also tested and, if significant, a post hoc analysis (Bonferroni's) was performed to check for between-study group differences at 3- and 6-month follow-up. For the continuous outcomes measured only at baseline and at 6 months, an unpaired t-test was conducted on the change from the baseline. Outcomes of categorical parameters were evaluated by binomial logistical regression with baseline values and study group as covariates. Exacerbations were analysed with a zeroinflated Poisson regression model. Adherence based on prescription refill was analysed using an unpaired t-test. A two-tailed significance level of 0.05 was used.

\section{RESULTS}

From January 2006 to April 2006, potentially eligible patients were identified at the participating pharmacies. The flow of participants through the study is shown in figure 1 . Of the 356 eligible patients, 155 were excluded before randomisation. The remaining 201 patients were randomly allocated to either the control or intervention group. Both study groups were well matched with regard to demographic and clinical characteristics (table 2).

\begin{tabular}{|c|c|c|}
\hline & Control group & Intervention group \\
\hline Subjects n & 94 & 107 \\
\hline Male sex & $46(49)$ & $48(45)$ \\
\hline Age yrs & $36.3(17-51)^{\#}$ & $35.2(19-51)^{\#}$ \\
\hline$B M I \mathbf{k g} \cdot \mathbf{m}^{-2}$ & $24.7(16.9-38.6)$ & $24.8(15.7-41.4)$ \\
\hline \multicolumn{3}{|l|}{ Education \% } \\
\hline No high school degree & 5.3 & 1.9 \\
\hline High school degree & 48.9 & 50.5 \\
\hline Higher education" & 44.7 & 47.7 \\
\hline \multicolumn{3}{|l|}{ Smoking status \% } \\
\hline Current smoker & 21.3 & 23.4 \\
\hline Ex-smoker & 29.7 & 20.7 \\
\hline Passive smoker & 30.7 & 29.4 \\
\hline Pack-yrs of current smokers & $5.9(0.1-10.0)$ & $5.5(0.5-10.0)$ \\
\hline Pack-yrs of ex-smokers & $4.0(0.1-10.0)$ & $5.6(0.3-14.0)^{\#}$ \\
\hline Asthma duration yrs & $22(1-48)$ & $20(1-47)$ \\
\hline Allergic asthma ${ }^{+} \%$ & 93.5 & 81.3 \\
\hline Tree & 47.1 & 51.7 \\
\hline Grass & 62.1 & 59.8 \\
\hline Dog & 41.4 & 42.5 \\
\hline Cat & 65.5 & 52.9 \\
\hline Dust mite & 87.4 & 82.8 \\
\hline Medication & 14.9 & 12.6 \\
\hline Asthma action plan \% & 34.8 & 33.6 \\
\hline \multicolumn{3}{|l|}{ Peak expiratory flow } \\
\hline Morning L $L \cdot \mathrm{min}^{-1}$ & 390.7 (127.9-755.0) & $409.7(165.7-717.1)$ \\
\hline Evening $L \cdot \mathrm{min}^{-1}$ & 409.7 (130.9-786.2) & $422.5(143.1-715.4)$ \\
\hline ACT score & $19.3(10-25)$ & $19.7(11-25)$ \\
\hline ACT $<15 \%$ of patients & 8.5 & 5.6 \\
\hline АСТ $15-19 \%$ of patients & 42.6 & 41.1 \\
\hline ACT $20-25 \%$ of patients & 48.9 & 53.3 \\
\hline Nights with awakenings \% & $9.4(0-100)$ & $7.4(0-100)$ \\
\hline $\begin{array}{l}\text { Rescue medication } \\
\text { puffs } \cdot \text { day }^{-1}\end{array}$ & $1.33(0-15.6)$ & $1.24(0-10.7)$ \\
\hline \multicolumn{3}{|l|}{ Controller medication \% } \\
\hline ICS & 23.1 & 25.0 \\
\hline LABA & 9.2 & 14.5 \\
\hline ICS + LABA combination & 70.8 & 64.5 \\
\hline Theophylline & 12.3 & 15.8 \\
\hline Leukotriene modifiers & 1.5 & 0.0 \\
\hline Mean daily dose of $\mathrm{ICS}^{\S} \mu \mathrm{g}$ & $1211.0(200-4000)$ & $1184.1(200-4000)$ \\
\hline
\end{tabular}

Data are presented as $\mathrm{n}(\%)$ or mean (range), unless otherwise indicated. For the parameters recorded on diary cards, the mean values of the 2 weeks of the run-in period were considered for baseline. Values did not differ significantly among both groups according to the unpaired t-test for continuous variables and the Chi-squared test for categorical variables. BMI: body mass index; ACT: Asthma Control Teste (QualityMetric, Lincoln, RI, USA); ICS: inhaled corticosteroids; LABA: long-acting $\beta_{2}$-agonists. ${ }^{\#}$ : deviation from inclusion criteria (included in al statistical analyses) was $n=1$ for "age" in the control group and in the intervention group, $\mathrm{n}=2$ for "pack-yrs of ex-smokers" in the intervention group; ": succeeded any type of higher education (college or university); ${ }^{+}$: self-reported allergy (via questionnaire); : : expressed as beclomethasone equivalent. 


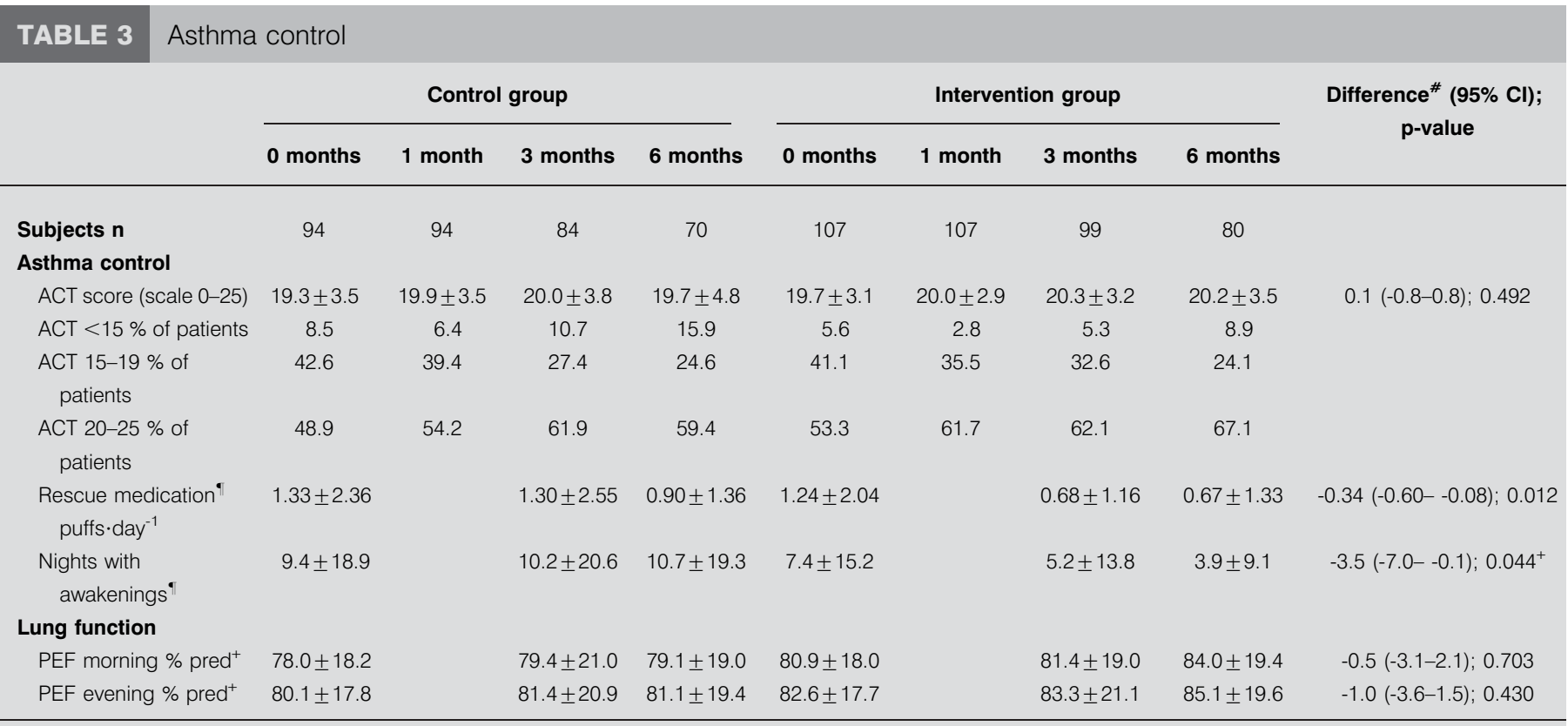

Data are presented as mean $\pm \mathrm{SD}$, unless otherwise indicated. Bonferroni's post hoc test showed no significant between-group difference at 3-month follow-up $(\mathrm{p}=0.529)$

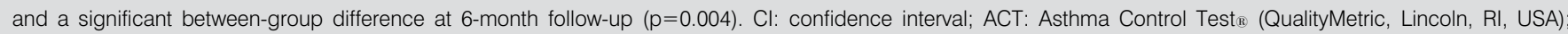
PEF: peak expiratory flow; \% pred: \% predicted. " : difference in mean change from baseline between intervention and control group ("intervention effect"); ": average over the previous 14 days; ${ }^{+}$: there was a significant study group-time interaction $(p=0.033)$

Of the 201 randomised patients, $150(75 \%)$ completed the study. Completion rates did not differ significantly by study group ( $\mathrm{p}>0.05)$. Reasons for drop out were as follows: personal reasons (15), withdrawal of the pharmacist from study (two), relocation (two), lost to follow-up (27) and other reasons (five). Baseline characteristics of patients not completing the study did not differ significantly from patients completing the study $(\mathrm{p}>0.05)$.

\section{Asthma control}

Mean ACT scores did not change from baseline for both study groups (table 3). However, a pre-defined subgroup analysis of patients having insufficiently controlled asthma at baseline showed that the intervention had significantly increased the ACT score after 6 months compared with usual care (mean ACT change from baseline in the intervention group was +2.3 and +0.3 in the control group; mean difference $(95 \%$ confidence interval), 2.0 (0.1-3.9); $\mathrm{p}=0.038$; fig. 2).

The need for rescue medication was reduced in both groups from baseline, with a significantly higher reduction in the intervention arm (-0.56 and -0.57 inhalations per day after 3- and 6-month follow-up, respectively) versus the control arm (-0.03 and -0.43 inhalations per day after 3- and 6-month follow-up, respectively; $p=0.012$ ). Patients in the intervention group experienced less night-time awakenings due to asthma than patients in the control group ( $p=0.044$; table 3$)$. For this outcome measure, there was a significant interaction between study group and time $(\mathrm{p}=0.033)$. Post hoc analysis showed that the intervention group had significantly fewer nightly awakenings than the usual care group at 6-month follow-up $(p=0.004)$, while there was no difference at 3 -month follow-up $(p=0.529)$.
The present study found no within-subject or between-group differences for the PEF morning and PEF evening values (table 3).

\section{Severe asthma exacerbations}

The present study found no differences between the control and intervention groups in the occurrence of severe exacerbations (table 4).

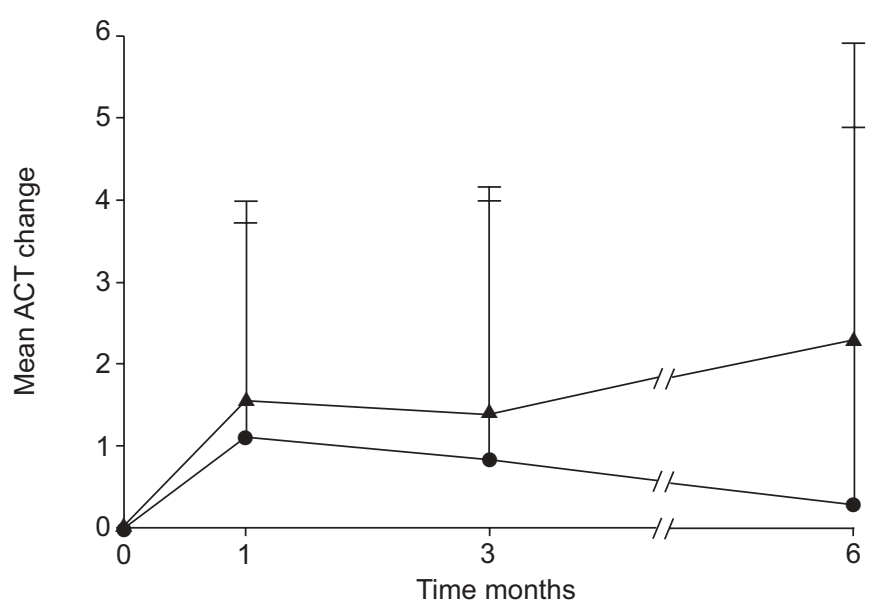

FIGURE 2. Mean change \pm SD from baseline in Asthma Control Test (ACT; QualityMetric, Lincoln, RI, USA) score for a pre-defined subgroup of patients having an ACT score <20 ("insufficiently controlled asthma"). The intervention group

) is compared at baseline with the control group (A) 


\section{TABLE 4 Severe exacerbations}

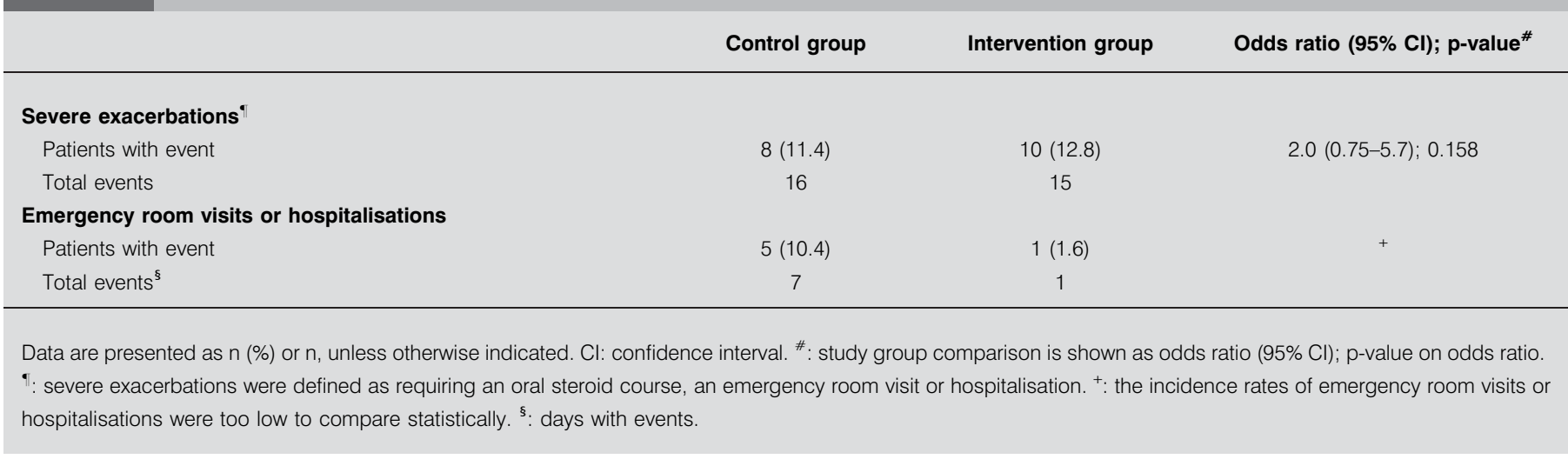

\section{Adherence to controller medication}

Adherence to controller medication during the course of the study, as judged by the prescription refill rates, was higher in the intervention group compared with the control group (mean adherence rate 90.3 versus $74.6 \% ; p=0.016$ ). However, there was no significant between-group difference in medication adherence as assessed by self-reporting $(p=0.108$; table 5$)$.

\section{Asthma-specific quality of life}

The present study found no significant difference in Asthma Quality of Life Questionnaire (AQLQ) score between both study groups, either at baseline or at the end of the follow-up period (table 5).

\section{Inhalation technique and knowledge on asthma}

At baseline, the mean percentage of handling steps performed correctly was $\sim 75 \%$ in both groups (table 5 ). At the end of follow-up, this percentage was significantly higher in the intervention arm $(p=0.004)$. The percentage of patients performing each of the inhalation manoeuvres correctly increased by $40 \%$ in the pharmacist care group and by $20 \%$ in the usual care group. The intervention was also able to

\section{TABLE 5 Other secondary outcomes}

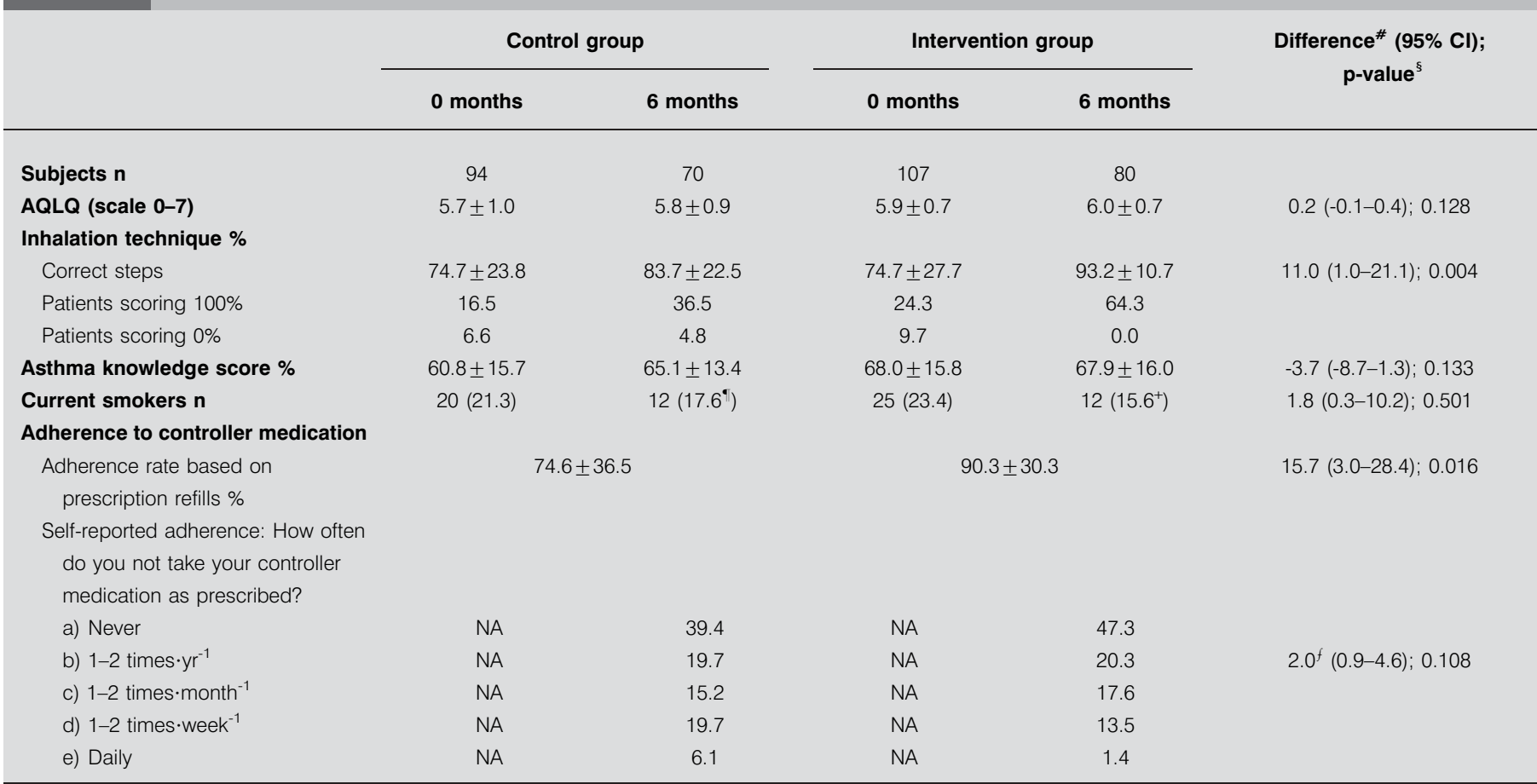

Data are presented as mean $\pm \mathrm{SD}$ or $\mathrm{n}(\%)$, unless otherwise indicated. Cl: confidence interval; AQLQ: Asthma Quality of Life Questionnaire; NA: not assessed. \#: difference in mean change from baseline between intervention and control group ("intervention effect"), except for adherence, which was assessed during the entire study period (based on prescription refill) and at 6 months only (based on self-report). " : percentage was calculated for 68 patients (two missing values). ${ }^{+}$: percentage was calculated for 77 patients (three missing values). s. odds ratio $(95 \% \mathrm{Cl})$; p-value on odds ratio. $f$ : for statistical analysis of self-reported adherence, the answers were regrouped into two categories: "adherent" group = answers a)-c) and "nonadherent" group = answers d) and e). 
correct all major inhalation technique errors, as $9.7 \%$ of the patients were assigned a sum score of zero before the intervention, a percentage reduced to $0.0 \%$ at the end of the intervention period. For patients receiving usual care, these percentages were $6.6 \%$ (at the start of the study) and $4.8 \%$ (at the end of the study). No beneficial effects of the intervention were seen in the asthma knowledge scores (table 5).

\section{Smoking}

At the start of the study, $20(21.3 \%)$ patients in the control group and $25(23.4 \%)$ patients in the intervention group reported to be current smokers. Of the smoking patients in the control group, two had quit smoking, 12 were still smoking and six were lost to follow-up after 6 months. Of the smokers in the intervention group, four had quit smoking, 12 were still smoking and nine were lost to follow-up after 6 months. No significant between-group differences were observed (table 5).

\section{DISCUSSION}

The present article reports on the first randomised controlled trial assessing the impact of a community pharmacist intervention promoting optimal asthma medication use on asthma control. Importantly, in accordance with GINA 2006 guidelines, this pharmacist intervention was specifically tailored to the patient's current asthma control. The current authors found that their programme substantially improved both the inhalation technique and medication adherence, which are both key stones for successful asthma management. The intervention also improved asthma control of insufficiently controlled patients and, for the entire study group, reduced the use of reliever medication and the frequency of nocturnal awakenings. It seems likely that these clinical improvements result from the more appropriate use of the asthma controller medication.

Although correct use of the inhaler device is essential for the medicine to arrive at its target organ, i.e. the lower airways, its importance is often overlooked. There is evidence that poor inhaler technique is associated with poor asthma control [27-29]. Recently, the Aerosol Drug Management Improvement Team demonstrated that many patients do not use their inhaler devices correctly, and therefore strongly urged healthcare workers to be more aware of this major problem [30]. Community pharmacists could play an important role in this area, by teaching the patients how to use their inhaler devices properly and regularly checking the technique during the course of treatment (especially when therapeutic goals are not met).

Besides inhalation technique, effective asthma management also depends on the patient's adherence to the prescribed controller medication. Several studies have shown that adherence to chronic asthma therapy is low, mainly with respect to inhaled corticosteroids [8, 31]. Nevertheless, previous studies have demonstrated that regular use of inhaled corticosteroids may reduce asthma-related hospitalisation and death $[32,33]$. In the present study, almost all patients were prescribed inhaled corticosteroids as controller treatment. Patients in the intervention arm were $\sim 15 \%$ more adherent than patients receiving usual care (according to the prescription refill data, which are a more objective measure of adherence than self-reporting), suggesting a beneficial impact intervention. The present findings stress the importance of patient education about the necessity of inhaled corticosteroids as a way to improve adherence. For a correct interpretation of the results of the present study, it should be emphasised that the medication profiles, i.e. type and daily dose of asthma controller medication, remained unchanged during the study period in both groups (data not shown). This suggests that the improvements in symptom control seen in the intervention group can be ascribed to the extra pharmacist care and not to changes in pharmacotherapy.

Within the 6-month follow-up period, the presently discussed programme did not affect the patients' knowledge about asthma. A plausible explanation could be the fact that information about asthma was given briefly and was limited to the first visit (table 1, session 1), which is likely to be insufficient to significantly improve the knowledge of patients. Likewise, the asthma-related quality-of-life scores (already high at baseline in both groups) remained unchanged. Also, the intervention did not influence the occurrence of severe asthma exacerbations during the study period.

The overall present results are consistent with those of other community pharmacy-based programmes [10-15]. However, some of these previous studies have shown significant improvements in quality of life, peak flow values and asthma knowledge, while this was not found in the present study. A detailed overview of the results for these outcomes of the previous studies is shown in table 6. Regarding quality of life, the results of these studies are quite heterogeneous. This may be attributed to the use of a variety of quality-of-life instruments. Only one of these studies [15] used the AQLQ [24], which was also used in the present study. That study [15] also found no significant improvement in the quality-of-life scores. Moreover, patients in the present study already had high scores at baseline, reducing their room for improvement. For PEF, the studies reporting significant improvements were studies in which the PEF measurements were only performed in the intervention group, not in the control group. Thus, the reported improvements are "within-group" comparisons (prepost), not "between-group" comparisons (intervention group versus control group), with the exception of the study conducted by WEINBERGER et al. [15], which reported significantly higher PEF rates in the intervention group than in the control group after a follow-up period of 12 months (the present study lasted only 6 months). Four of the six previous studies assessed asthma knowledge: all of them showed a significant improvement (two of them after 6-month follow-up, and two after 12-month follow-up). A possible explanation for the fact that improved knowledge was not observed in the present study has already been provided in the present article, namely the limited information about asthma provided during intervention.

The present study has two important assets compared with the previously published trials, as follows. 1) It was the first to investigate the effectiveness of patient education using asthma control as the primary outcome. 2) An intervention was tested that was specifically tailored to the current asthma control status of the patient, concordant with the new GINA asthma management guidelines. The ACT was shown to be an excellent tool to measure rapidly [17] and accurately [20] the asthma control of patients presenting at community 
TABLE 6 A detailed overview of the results regarding quality of life, peak expiratory flow and knowledge of the previously published trials of community pharmacy-based programmes of asthma care

\begin{tabular}{|c|c|c|c|}
\hline First author [ref.] & $\begin{array}{c}\text { Duration } \\
\text { months }\end{array}$ & Results & Comments \\
\hline ARMOUR [10] & & PEF: NA & $\begin{array}{l}\text { No PEF assessed. FEV1 and FEV1/FVC were } \\
\text { assessed; however, no significant improvements } \\
\text { were observed }\end{array}$ \\
\hline \multirow{2}{*}{ BARBANEL [11] } & & PEF: NA & \\
\hline & & Knowledge: NA & \\
\hline \multirow[t]{2}{*}{ MANGIAPANE [12] } & 12 & $\begin{array}{l}\text { QoL: significantly improved within the } \\
\text { intervention group }\end{array}$ & $\begin{array}{l}\text { Study was not a randomised controlled trial, but it } \\
\text { had a pre-post design (without concurrent control } \\
\text { group); consequently, no intervention versus } \\
\text { control group comparison was possible }\end{array}$ \\
\hline & & $\begin{array}{l}\text { Knowledge: significantly improved within the } \\
\text { intervention group }\end{array}$ & \\
\hline \multirow[t]{3}{*}{ SAINI [13] } & 6 & QoL: significant improvement & $\begin{array}{l}\text { PEF was only measured in the intervention group } \\
\text { (not in the control group), so no intervention versus } \\
\text { control group comparison was possible }\end{array}$ \\
\hline & & $\begin{array}{l}\text { PEF: significantly improved within the } \\
\text { intervention group }\end{array}$ & \\
\hline & & Knowledge: significantly improved & \\
\hline \multirow[t]{2}{*}{ Schulz [14] } & 12 & QoL: significantly improved & $\begin{array}{l}\text { PEF was only measured in the intervention group } \\
\text { (not in the control group), so no intervention versus } \\
\text { control group comparison was possible }\end{array}$ \\
\hline & & $\begin{array}{l}\text { PEF: PEF morning remained status quo, PEF } \\
\text { evening was significantly improved within the } \\
\text { intervention group }\end{array}$ & \\
\hline
\end{tabular}

QoL: quality of life; PEF: peak expiratory flow; NA: not assessed; FEV1: forced expiratory volume in one second; FVC: forced vital capacity.

pharmacies, enabling the pharmacist to provide each patient with specific advice tailored to his/her clinical needs.

The present investigation also has its limitations. First, the effect of the intervention was probably underestimated, as newly diagnosed, steroid-naïve asthma patients were not included, but only patients who had already been taking chronic asthma medication for $\geqslant 1 \mathrm{yr}$. In fact, although all patients in the present study had already been on treatment for $\geqslant 1 \mathrm{yr}$, the present programme was still beneficial. It is likely that the intervention will have a greater impact on treatmentnaïve asthma patients, as they have a higher need for information and training about optimal asthma medication use. For safety reasons, patients with seriously uncontrolled asthma (ACT <15) were excluded from participation. This generated a study population with a limited potential for improvement, which may also have caused an underestimation of the impact of the intervention. Secondly, patients in the present study may not be fully representative of the overall general population of asthma patients, since they participated voluntarily in the study. Moreover, only regular pharmacy customers were recruited in order to ensure sufficient followup during the course of the study. This selected patient sample may reflect those with a stronger interest in self-management, possibly generating a positive selection bias. Thirdly, randomisation was performed on a patient level, meaning that each pharmacy had to follow up control subjects, as well as intervention patients. To prevent contamination between both study groups, participating pharmacists were thoroughly instructed on how to strictly follow the protocol prior to onset of the trial. Fourthly, the present study was an open study, in which data collection was performed by the person who also 
delivered the intervention. Nevertheless, to prevent bias, the final data collection (i.e. after 6-month follow-up) for all outcomes was performed by a pharmacist other than the pharmacist who administered the intervention. Lastly, the sustainability of beneficial outcomes beyond 6 months was not assessed. However, the clear differences between intervention and control group argue for further investigation and largerscale implementation.

The present study suggests that rigorously designed trials (conforming to the Consolidated Standards of Reporting Trials statement [34]) evaluating realistic interventions in real clinical practice settings are of paramount importance for asthma control improvement, especially in primary care. Community pharmacists are well placed in the healthcare system to provide patients with education on their asthma medication, as they have the advantages of easy patient access and regular patient contact. The brief, simple and pragmatic intervention carried out in the present study is feasible to implement in all community pharmacies, which argues for the generalisability of the present results. Moreover, the random selection of the pharmacies participating in the present study, and the community-based patient selection using fairly straightforward inclusion criteria also pleads for the generalisability of the study results [35].

In conclusion, the authors would like to stress that this pharmacist intervention is not meant to replace formal asthma education but rather to complement it. The clear need for patient-focused care on appropriate use of asthma medication has already been highlighted $[6-9,30]$ and it is an essential strategy to improve asthma control, especially in primary care. Further research should focus on the cost-effectiveness of such interventions.

\section{ACKNOWLEDGEMENTS}

The authors would like to thank the participating patients and pharmacists. They also thank GlaxoSmithKline (GSK) Belgium for their permission to use the Asthma Control Test ${ }$. The authors are grateful to K. Van Steen and P. Konings (Biostatistics, Ghent University Hospital, Ghent, Belgium) for their statistical advice.

\section{REFERENCES}

1 Pauwels RA, Lofdahl C-G, Postma DS, et al. Effect of inhaled formoterol and budesonide on exacerbations of asthma. N Engl J Med 1997; 337: 1405-1411.

2 Rabe KF, Adachi M, Lai CK, et al. Worldwide severity and control of asthma in children and adults: the global asthma insights and reality surveys. J Allergy Clin Immunol 2004; 114: 40-47.

3 Global Initiative for Asthma. Global strategy for asthma management and prevention, 2006. www.ginasthma.org. Date last updated: July 20, 2007. Date last accessed: November 19, 2007.

4 Global Initiative for Asthma (GINA). Global strategy for asthma management and prevention. NHLBI/WHO workshop report. National Institutes of Health, National Heart, Lung and Blood Institute. Bethesda, National Institutes of Health, 1995; publication no. 95-3659.
5 Global Initiative for Asthma. Global strategy for asthma management and prevention. NHLBI/WHO workshop report. National Institutes of Health, National Heart, Lung and Blood Institute. Bethesda, National Institutes of Health, 2002; publication no. 02-3659.

6 Horne R. Compliance, adherence, and concordance: implications for asthma treatment. Chest 2006; 130: Suppl. 1, 65S-72S.

7 Boulet LP. Perception of the role and potential side effects of inhaled corticosteroids among asthmatic patients. Chest 1998; 113: 587-592.

8 Cochrane MG, Bala MV, Downs KE, Mauskopf J, BenJoseph RH. Inhaled corticosteroids for asthma therapy: patient compliance, devices, and inhalation technique. Chest 2000; 117: 542-550.

9 Laforest L, Van Ganse E, Devouassoux G, et al. Influence of patients' characteristics and disease management on asthma control. J Allergy Clin Immunol 2006; 117: 1404-1410.

10 Armour C, Bosnic-Anticevich S, Brillant M, et al. Pharmacy Asthma Care Program (PACP) improves outcomes for patients in the community. Thorax 2007; 62: 496-502.

11 Barbanel D, Eldridge S, Griffiths C. Can a self-management programme delivered by a community pharmacist improve asthma control? A randomised trial. Thorax 2003; 58: 851-854.

12 Mangiapane S, Schulz M, Muhlig S, Ihle P, Schubert I, Waldmann HC. Community pharmacy-based pharmaceutical care for asthma patients. Ann Pharmacother 2005; 39: 1817-1822.

13 Saini B, Krass I, Armour C. Development, implementation, and evaluation of a community pharmacy-based asthma care model. Ann Pharmacother 2004; 38: 1954-1960.

14 Schulz M, Verheyen F, Muhlig S, et al. Pharmaceutical care services for asthma patients: a controlled intervention study. J Clin Pharmacol 2001; 41: 668-676.

15 Weinberger M, Murray MD, Marrero DG, et al. Effectiveness of pharmacist care for patients with reactive airways disease: a randomized controlled trial. JAMA 2002; 288: 1594-1602.

16 Van Mil JW, Tromp TF. European barriers to the implementation of pharmaceutical care. Int J Pharm 2001; 9: 163-168.

17 Mehuys E, Van Bortel L, Annemans L, et al. Medication use and disease control of asthmatic patients in Flanders: a cross-sectional community pharmacy study. Respir Med 2006; 100: 1407-1414.

18 Global Initiative for Asthma Patient Guide. Wat U en Uw Familie aan Astma Kunnen Doen [What You and Your Family Can Do About Asthma]. National Institutes of Health. Bethesda, National Institutes of Health (NIH), 1995; publication no. 96-3659C.

19 Nathan RA, Sorkness CA, Kosinski M, et al. Development of the asthma control test: a survey for assessing asthma control. J Allergy Clin Immunol 2004; 113: 59-65.

20 Schatz M, Sorkness CA, Li JT, et al. Asthma Control Test: reliability, validity, and responsiveness in patients not previously followed by asthma specialists. J Allergy Clin Immunol 2006; 117: 549-556.

21 Radeos MS, Camargo CA Jr. Predicted peak expiratory flow: differences across formulae in the literature. $A m J$ Emerg Med 2004; 22: 516-521. 
22 Osterberg L, Blaschke T. Adherence to medication. N Engl J Med 2005; 353: 487-497.

23 Hess LM, Raebel MA, Conner DA, Malone DC. Measurement of adherence in pharmacy administrative databases: a proposal for standard definitions and preferred measures. Ann Pharmacother 2006; 40: 1280-1288.

24 Juniper EF, Buist AS, Cox FM, Ferrie PJ, King DR. Validation of a standardized version of the Asthma Quality of Life Questionnaire. Chest 1999; 115: 1265-1270.

25 World Health Organization and Danish College of Pharmacy Practice. Pharmacy-based asthma services: protocol and guidelines, 1998. www.euro.who.int/ Document/E67662.pdf. Date last updated: 1998. Date last accessed: November 19, 2007.

26 Beunckens C, Molenberghs G, Kenward MG. Direct likelihood analysis versus simple forms of imputation for missing data in randomized clinical trials. Clin Trials 2005; 2: 379-386.

27 Giraud V, Roche N. Misuse of corticosteroid metered-dose inhaler is associated with decreased asthma stability. Eur Respir J 2002; 19: 246-251.

28 Newman SP, Weisz AW, Talaee N, Clarke SW. Improvement of drug delivery with a breath actuated pressurised aerosol for patients with poor inhaler technique. Thorax 1991; 46: 712-716.
29 Lindgren S, Bake B, Larsson S. Clinical consequences of inadequate inhalation technique in asthma therapy. Eur $\mathrm{J}$ Respir Dis 1987; 70: 93-98.

30 Crompton GK, Barnes PJ, Broeders M, et al. The need to improve inhalation technique in Europe: a report from the Aerosol Drug Management Improvement Team. Respir Med 2006; 100: 1479-1494.

31 Cerveri I, Locatelli F, Zoia MC, Corsico A, Accordini S, de Marco R. International variations in asthma treatment compliance: the results of the European Community Respiratory Health Survey (ECRHS). Eur Respir J 1999; 14: 288-294.

32 Suissa S, Ernst P, Benayoun S, Baltzan M, Cai B. Low-dose inhaled corticosteroids and the prevention of death from asthma. N Engl J Med 2000; 343: 332-336.

33 Donahue JG, Weiss ST, Livingston JM, Goetsch MA, Greineder DK, Platt R. Inhaled steroids and the risk of hospitalization for asthma. JAMA 1997; 277: 887-891.

34 Moher D, Schulz KF, Altman DG. The CONSORT statement: revised recommendations for improving the quality of reports of parallel-group randomised trials. Lancet 2001; 357: 1191-1194.

35 Bonell C, Oakley A, Hargreaves J, Strange V, Rees R. Assessment of generalisability in trials of health interventions: suggested framework and systematic review. BMJ 2006; 333: 346-349. 